\title{
One-pot Manufacture of Nanoparticle-based Films in Aqueous Media via an Electric Field-Driven
}

Assembly Process

Isabel Gonzalo-Juan ${ }^{a}$, Juan A. Escribano ${ }^{a}$, Yolanda Castro $^{a}$, Antonio J. SanchezHerencia $^{a}$, Jose Luis G. Fierro ${ }^{b}$, and Begoña Ferrari ${ }^{a, *}$

${ }^{a}$ Instituto de Cerámica y Vidrio, CSIC, Kelsen 5, 28049 Madrid, Spain

${ }^{\mathrm{b}}$ Instituto de Catalisis y Petroleoquimica, Marie Curie 2, 28049 Madrid, Spain

igonzalo@icv.csic.es; $\quad$ jescribano@icv.csic.es;

ajsanchez@icv.csic.es; jlgfierro@icp.csic.es; bferrari@icv.csic.es

*Instituto de Cerámica y Vidrio, CSIC, Kelsen 5, 28049 Madrid, Spain; phone: +34 917355840; fax: +34 917355843; email: bferrari@icv.csic.es 


\begin{abstract}
Assembly of nanoparticles on multiple length scales and large areas is crucial for the manufacture of nanostructured devices. Nowadays, major problems in nanoparticle synthesis and their processing are the handling of waste (such as solvents, sub-products and unreacted precursors) and the search for environmental tolerable procedures. Environmental and economical pressures are now forcing the chemical community to search for more efficient ways of performing particles synthesis and their processing. Thus, it would be much more efficient if both processes synthesis and processing could be realized in one sequence without isolating the intermediates. Here, we detail the results of a sustainable approach in which synthesis and the as-synthesized nanoparticles assembly are realized in one sequence without any intermediate step such as drying, sieving or centrifuge. The one pot process proposed emphasized the synergism between the mild hydrothermal synthesis of yttrium stabilized zirconia (YSZ) nanoparticles and their processing based on an electric field-driven mechanism that provides the assembly of the as-synthesized nanoparticles suspended in the synthesis mother water with low solid loading $\left(<1 \mathrm{~g} \mathrm{~L}^{-1}\right)$. The layer-by-layer or Frankvan-der-Merwe growth of an extremely densely packed nanoparticle-based film consolidated by green technologies was highlighted. Results suggest a fast $(<1 \mathrm{~h})$ and reliable shaping process that could approach to a full yield. The approach is described for YSZ nanospheres, but it could be applicable to any nanoparticle and/or synthesis process offering a controlled, robust, inexpensive route for a large-scale manufacture of densely packed nanoparticle-based films. The key step of the process is the stabilization of the as-synthesized nanoparticle in the post-reaction medium using the synthesis catalyst itself, urea, and an additional dispersant, Polyethyleneimine (PEI). The presence of organic modifiers in a low concentration $(<2 \mathrm{wt} \%$ on the base of solids in the suspension) is critical and determines movement and re-arrangement of nanoparticles under the influence of an electric field during film growth.
\end{abstract}

\title{
Keywords
}

Nanoparticle Assembly, Electrophoretic Deposition, Wet Synthesis, Colloidal Processing, Thin films, Zirconia, Hydrothermal Synthesis. 


\section{Introduction}

Nanoparticles in electronic, optical and medical devices have made an impact in almost every aspects of daily life. The essential features of the global nanoparticle processing are nanoparticle synthesis and dispersion followed by their conformation. Despite the great success and the importance of nanoparticles chemistry to life quality, its public image has been deteriorated in the last two decades. Effective nanoparticle processing is predicated on site isolation, the physical separation of reagents and or catalyst from each other and frequently the as synthesized nanoparticles have been dried, freeze, milled, sieved and centrifuged before their processing. ${ }^{1-5}$ Nanoparticle synthesis and their processing typically achieve site isolation by using separate flacks or reactors. Iterative transformation and purification by using "multiple pots" has been a successful model, but it is plagued by wastes mostly manifested in the form of solvents. Beyond solvents, high-yielding reactions involving both synthesis and processing, often produced salts and other impurities that must be removed to avoid deleterious effects in the final devices. Nowadays, major problems in nanoparticle synthesis and their processing are the handling of waste and the search for environmental tolerable procedures. Environmental and economic pressures are now forcing the chemical community to search for more efficient ways of performing particles synthesis and their processing. Thus, it would be much more efficient if both processes synthesis and processing could be realized in one sequence without isolating the intermediates. Compared with stepwise process the amount of solvents, reagents, adsorbents and energy would be dramatically decreased and the amount of work would be reduced. Moreover, the one pot process can drive the equilibrium to the desire direction allowing an ecologically and economically production.

A one-pot process for the manufacture of dense YSZ nanoparticle films, combining the hydrothermal synthesis of these nanoparticles and their electrically-assisted assembly by ElectroPhoretic Deposition (EPD), is proposed in this work as an alternative to the selfassembly through evaporative processes. Opposite to them, in EPD, the forced assembly and packing of the as-synthesized nanoparticles under the influence of an electric field precedes the solvent evaporation or deposition. The immediate consequences of this fact are lower restrictions for solid loadings of suspensions and then, higher flexibility for 
nanostructure tailoring. Moreover, compared with the current state-of-the-art in nanoparticle film casting, the EPD has numerous and attractive characteristics, including film size scalability (from $100 \mathrm{~nm}^{2}$ to $1 \mathrm{~m}^{2}$ ), flexible shapes, superior assembly rates, and tightly-packed nanoparticle structures. ${ }^{3,6}$

Different kind of particles (oxides, non-oxides, metals, polymers, etc.) are suitable for EPD, which increases significantly its technological applicability as a Near Net Shaping (NNS) process for the sustainable manufacture of bulk items, thick and thin films or micropatterned arrays. Several reviews have highlighted the fundamentals of the EPD and its novel application as a tool to manipulate nanoscale entities and their assembly in macroscopically useful structures and devices. ${ }^{2,3,6,7}$ Enhancing the inherent capabilities of this process for the manipulation and deposition of particles, EPD and related electric field assisted deposition methods, can lead to the innovation on materials and devices fabrication. ${ }^{8,9}$

From a chemical standpoint, such processes suffer virtually no limitations. Notwithstanding, fine-tuning of the surface electrical properties for suspended particles 10, 11 and their subsequent compaction driven by an electrical force, could provide a universal method for the manipulation and placing of a large range of entities for many applications, such as nanocrystals, mesoporous nanoparticles or nano-dyes. Those techniques can therefore be used to meet a variety of challenges facing the materials science and technology community, i.e. the insertion and tailoring of ordered and nanosized structures through a conventional and easily scalable manufacture process. However, the complete and mutual integration of all chemical, physical and engineering aspects remains a challenging task. In a one-pot process, the challenges in the control of nanoparticle arrangement under the influence of an electric field must be met with the manipulation of colloidal chemistry of the as-synthesized nanoparticles. The control of the dispersion and the surface stability of nanoparticles suspended in the post-reaction medium require a deep knowledge of the chemical and physical mechanisms on the nanoparticle surfaces. In this work, one of our goals has been to optimize the colloidal conditions of the YSZ nanocrystals suspended in the post-reaction medium from the hydrothermal synthesis to achieve the consolidation of a dense YSZ film by EPD without any further drying, cleaning, and sieving or concentration process. 
Understanding of nanoparticle dynamics in polar and non-polar solvents is mandatory to control the nanoparticle arrangement in EPD. ${ }^{12,} 13$ More specifically, nanoparticleliquid, nanoparticle-nanoparticle and electric field-nanoparticle interactions must be addressed. In this scene, organic molecules, surfactants and co-polymers used as synthesis modifiers or dispersants not only play a key role during the electric-driven arrangement or during the chemical evolution of the surrounding ${ }^{14,15}$ and patterning ${ }^{16}$ electrode, but also in the activation and in the electrochemical affinity of the substrates. ${ }^{6}$

The one-pot process approach, regarded as synthesis, stabilization and EPD of nanoentities, has been proved for non-polar solvents ${ }^{17}$ and for a hybrid sol-gel synthesis route. ${ }^{18,19}$ In both cases, solvents diminished the effects of the water electrolysis during the deposition. The mechanism that we propose now is to process dense and entirely films by electrophoretic deposition using well dispersed as-synthesized nanoparticles hydrothermally synthesized at mild conditions, meaning at temperatures lower than 180 ${ }^{\circ} \mathrm{C}$. Moreover, both the synthesis and the EPD are performed in water which is the most universal, green and singular solvent considered. Finally, growth behavior and an unusual low temperature not higher than $500{ }^{\circ} \mathrm{C}$ for the film consolidation was determined.

\section{Materials and Methods}

Synthesis: YSZ nanoparticles (NPs) were synthesized under mild hydrothermal conditions $\left(\mathrm{T} \leq 180^{\circ} \mathrm{C}\right)$ as described previously by the authors ${ }^{20}$. Zirconyl nitrate, $\mathrm{ZrO}\left(\mathrm{NO}_{3}\right)_{2}\left(\mathrm{xH}_{2} \mathrm{O}\right)$ (MaTeck, Germany), and yttrium nitrate, $\mathrm{Y}\left(\mathrm{NO}_{3}\right)_{3}\left(\mathrm{xH}_{2} \mathrm{O}\right)(\mathrm{MaTeck}$, Germany) were used as zirconium and yttrium sources, and urea (Panreac, Spain) as pseudo-catalytic agent. Appropriate amounts of each precursor were employed to prepare the stock solutions of $5 \times 10^{-2} \mathrm{M}$ of $\mathrm{ZrO}_{2}{ }^{+2}$ and $5 \times 10^{-3} \mathrm{M}$ of $\mathrm{Y}^{3+}$ in distilled water at room temperature. $8 \times 10^{-2} \mathrm{M}$ of urea (Panreac, Spain) was employed as starting reagent. The stock solutions of the metal nitrides and urea were mixed at room temperature in adequate $\operatorname{ratios}^{20,21}$. After stirring for $1 \mathrm{~h}$, the mix solution was transferred into a $250 \mathrm{~mL}$ Teflon-lined reactor and heated at $80{ }^{\circ} \mathrm{C}$ for $24 \mathrm{~h}$ ( lower temperature step) and then at $180{ }^{\circ} \mathrm{C}$ for $48 \mathrm{~h}$ (higher temperature step). Total reflection X-ray fluorescence (TXRF spectrometer EXTRA-II, Rich \& Seifert, Germany) has been 
used for the elemental quantitative determination of $\mathrm{Zr}$ and $\mathrm{Y}$ dissolved in the mother water after synthesis (post-reaction medium). The as-synthesized YSZ nanoparticles were dispersed within the post-reaction medium by sonication (Ultrasonication Probe, UP 400S, Hielscher, Germany) and later stabilized adding 1 wt.\% of polyethyleneimine (PEI, Mw = 25000, Sigma Aldrich Co., USA.

EPD: Cathodic EPD was performed to deposit YSZ nanoparticles onto electropolished steel AISI 304. A platinum foil was used as counter-electrode. The electrodes were mounted in a vertical parallel-plate configuration with a gap of $2 \mathrm{~cm}$. Current densities $<200 \mu \mathrm{A} \mathrm{cm}^{-2}$, which correspond to electrical fields $<1.5 \mathrm{~V} \mathrm{~cm}^{-1}$, were applied in each experiment from 0 to $60 \mathrm{~min}$.

Characterization: Specific surface $\left(S_{\mathrm{s}}\right)$ and density $(\rho)$ of the as-synthesized nanoparticles were performed using $\mathrm{N}_{2}$ adsorption- BET method (Monosorb Surface Area Analyser MS-13, Quantachrome Co., USA) and helium pycnometry (Multipycnometer, Quantachrome Co., USA), respectively. X-ray photoelectron spectroscopy (XPS) spectra were carried out on Fisons Escalab 200R spectrometer using Mg Ka X-ray (photon energy, h.v = $1253.6 \mathrm{eV}$ ) source. The spectra were recorded in the constant pass energy mode $(20 \mathrm{eV})$. A survey spectrum was recorded for each sample (single scan) followed by detailed analysis (60 scans) of $\mathrm{Zr} 3 \mathrm{~d} 5 / 2$, Y 3d5/2, and $\mathrm{O} 1 \mathrm{~s}$ energy regions. The binding energies (BEs) were referenced to the spurious $\mathrm{C}$ 1speak $(284.6 \mathrm{eV})$ used as internal standard. The accuracy of quantitative analysis is $4 \%$ and the precision for the $\mathrm{BE}$ is $\pm 0.1 \mathrm{eV}$. The areas of the peaks were computed by fitting the experimental spectra to Gaussian/Lorentzian curves after removal of the background (Shirley function). Surface atom ratios were calculated from peak area ratios normalized by the corresponding atomic sensitivity factors ${ }^{22}$. High resolution transmission electron microscopy (TEM) images were performance on JEOL JEM-2011 microscope at an accelerating voltage of $200 \mathrm{kV}$. Powder X-Ray Diffraction measurements were performed on a D5000, Siemens diffractometer $\left(\mathrm{Cu} \mathrm{K} \alpha_{1}: 0.154056\right.$ $\mathrm{nm})$. The data were collected in the $2 \theta$ range from $20^{\circ}$ to $80^{\circ}$ at a step scan rate of $2^{\circ} \mathrm{s}^{-1}$. For the crystallographic phase determination, the observed peaks were processed using the ASTM- Files 37-1484, 48-0224 and 82-1246 for monoclinic, tetragonal and cubic phases, respectively. We confirmed the crystallographic phases detected by XRD by performing FT-Raman spectroscopy $\left(\lambda=\right.$ red $\mathrm{nm}$, Renishaw). Dried $\left(60^{\circ} \mathrm{C}\right)$ and calcined 
$\left(300^{\circ} \mathrm{C}\right)$ nanocrystals were characterized by attenuated total reflectance (ATR) Fourier Transform infrared spectroscopy (FTIR) using a Nicolet 20SXC Spectrometer. Thermal characterization of the as-synthesized YSZ powders was performance using a thermogravimeter analyzer (ATD-TG, STA 409, Netzsch, Germany) with a heating rate of 5 ${ }^{\circ} \mathrm{C} \mathrm{min}^{-1}$. The hydrodynamic size distribution of the as synthesized nanoparticles suspended in the mother liquor were measured on Zetasizer Nano ZS system. The nanoparticles suspended in the mother liquor were diluted to fit the accurate concentration for the size measurements.

The morphology of the as-deposited coatings was determined by Atomic Force Microscopy (AFM) on a Cervantes AFM System (Nanotec Electronica, S.L., Spain) operating in non-contact dynamic mode with amplitude modulation. Silicon nitride tips with a radius lower than $7 \mathrm{~nm}$ were used. The tips have a resonance frequency near to $330 \mathrm{kHz}$ and a force constant in the range of $42 \mathrm{~N}^{-m^{-1}}$. All AFM images were taken in air at room temperature. The AFM piezoelectric allows a maximum sample XY scan of $10 \times 10 \mu \mathrm{m}^{2}$. In order to obtain representative information from the films surfaces at least three separate areas of each film were measured, dynamic mode parameters were managed for a better resolution avoiding lateral effects of tip-sample interactions.

The electrophoretically deposited coatings were treated at $500{ }^{\circ} \mathrm{C}$ for $30 \mathrm{~min}$ with heating and cooling rates of $5{ }^{\circ} \mathrm{C} \mathrm{min}^{-1}$ to force the consolidation of the YSZ microstructure. Some samples were bended to qualitatively determine the degree of the film consolidation and density. The system (coating/substrate) was characterized by field emission scanning electron microscopy (FE-SEM) in a S-4700 microscope (Hitachi, Japan).

Ellipsometry, UV-Vis transmittance and, Instrumented Indentation of both the asdeposited and the annealed films were performed. Ellipsometry measurements were carrying out using a Spectral Ellipsometer (M-2000UTM, J.A. Co., Woollam. The spectra were taken within the spectral region $250-900 \mathrm{~nm}$ at a fixed incident angle of $75^{\circ}$. The data were fitted using the WVASE32 software using the Lorentz oscillator model. The refractive index (n) (taken at $\lambda=700 \mathrm{~nm}$ ) and the thickness (d) of the films were estimated from the fitted data.

To characterize the optical properties, transmittance spectra were acquired at room temperature in the UV-Vis spectral range (l= $1500-200 \mathrm{~nm})$ using a Lambda 950- 
Perkin Elmer spectrophotometer. Using the integrating sphere measurements were taken at different angles $\left(8^{\circ}, 25^{\circ}, 40^{\circ}\right.$ and $\left.68^{\circ}\right)$ with a step increment of $1 \mathrm{~nm}$.

Instrumented Indentation was performed using a CETR nanoindenter with Micro Module MH-2 and a Berkovich diamond tip. The applied load was $0.1 \mathrm{mN}$. Hardness and elastic modulus were calculated from the load vs displacement curve using the discharging of the maximum load. At least 3 indentations were employed to determine both the hardness and elastic modulus.

\section{Results and Discussion}

Figure 1 shows an overview of the one pot process studied in this work. It is made up of three steps: (step 1) the mild-hydrothermal synthesis of YSZ NPs $\left(\mathrm{T}<190^{\circ} \mathrm{C}\right)$ where urea plays an important role as pseudo-catalyst and stabilized reagent of both nanoparticles (as a surfactant) and medium (as pH regulator); the NPs suspended in the mother water of the synthesis are aggregated/agglomerated hence, (step 2) they are dispersed using ultrasound force (US) in order to brake the agglomerated NPs down, and then, stabilized by an electro-steric mechanism using PEI; the last step (step 3) corresponds to the film shaping: the stabilized nanoparticles are arranged as coatings by electrophoresis movement and electric-driven assembly.

The hydrothermal synthesis proposed in this work yields $0.56 \mathrm{gL}^{-1}$ of YSZ NPs suspended in the mother water. The $\mathrm{Zr}(\mathrm{IV})_{\mathrm{ac}}$ concentration in the post reaction medium determined by TXRF was $0.143 \mathrm{~mol} \%$, and consequently the reaction yield was $>98 \%$. Figure 2 shows an overview of the as-synthesized nanocrystals under mild conditions $\left(<180{ }^{\circ} \mathrm{C}\right)$ using an urea: $(\mathrm{ZrO})^{+2}$ ratio $(1: 3)$. Figures $2 \mathrm{a}$ and $2 \mathrm{c}$ depict an ensemble of the as-synthesized nanocrystals with $7.3 \pm 1.5 \mathrm{~nm}$ primary diameter sizes $\left(\mathrm{d}_{\text {TEM }}\right)$. The magnified image of them, figure $2 \mathrm{~b}$, shows a highly crystalline structure with spherical shape. The lattice fringes indexed was $0.286 \pm 0.003 \mathrm{~nm}$ which corresponds to the111 plane of 8YSZ (ASTM 30-1468). The X-ray diffraction (XRD) (figure 3a) along with the Raman spectrum analysis (figure $3 b$ ) of the as-synthesized nanocrystals indicated that the cubic phase is the main phase. Only a residual fraction of monoclinic, nondetectable by XRD, were detected by Raman.

Relevant features of the as-synthesized NPs after drying at $60{ }^{\circ} \mathrm{C}$ for $24 \mathrm{~h}$ were summarized in table I. The hydrothermal synthesis yield YSZ NPs with lower density 
than the theoretical density of YSZ $\left(\rho_{\mathrm{th}}=6.01 \mathrm{gcm}^{-3}\right)$ but, with a high specific surface $\left(\mathrm{S}_{\mathrm{s}}=140 \mathrm{~m}^{2} / \mathrm{g}\right)$. X-ray photoelectron spectroscopy (XPS) analysis was also studied. Table I summarizes the collected data from the $\mathrm{Zr} 3 d$ and $\mathrm{O} 1 s$ core-level XPS spectra (Figures $4 \mathrm{a}$ and $4 \mathrm{~b}$, respectively) of the as-synthesized nanoparticles. The binding energy (BE) of the most intense peak of the $\mathrm{Zr} 3 \mathrm{~d}$ doublet, $\mathrm{Zr} 3 \mathrm{~d} 5 / 2$, is the fingerprint of $\mathrm{Zr}^{4+}$ ions surrounded by oxide ions, while the $\mathrm{BE}$ contribution of $\mathrm{O} 1 \mathrm{~s}$ spectra (close to $530.0 \mathrm{eV})$ evidences the presence of the oxide moiety $(\mathrm{Zr}-\mathrm{O}-\mathrm{Zr})$. The high energy signal of the $\mathrm{O} 1 \mathrm{~s}$ profile (close to $531.0 \mathrm{eV}$ ) is related to the presence of $\mathrm{O}-\mathrm{H}$ groups bonded to terminal $\mathrm{Zr}^{4+}$ ions. ${ }^{23}$ The percentage of $\mathrm{O} 1 \mathrm{~s}$ peak linked to the metal (M-O) was higher than the percentage of $\mathrm{O}$ linked to the hydrogen $(\mathrm{H}-\mathrm{O})$, verifying that the mild hydrothermal method provides nanocrystals that are oxides without any extra thermochemical treatment. Moreover, the Y/Zr atomic ratio obtained from the XPS analysis for the as-synthesized nanoparticles is within the range of the $\mathrm{Y} / \mathrm{Zr}$ theoretical ratio calculated for $\mathrm{YSZ}, \mathrm{Y} / \mathrm{Zr}=16 / 92=0.174$, corroborating that the as-synthesized NPs have a cubic phase in agreement with HR-TEM, XRD and Raman analysis. An FTIR study of the as-synthesized NPs provides more information about them surfaces. Figure 5 shows the FTIR spectra of the as-synthesized NPs, the NPs calcined at $300^{\circ} \mathrm{C}$ for $30 \mathrm{~min}$ and urea. The spectrum of the urea $\left({ }_{2} \mathrm{HN}-\mathrm{CO}-\mathrm{NH}_{2}\right)$ exhibits a series of characteristic peaks of this organic molecule head groups vibrations at $3431 \mathrm{~cm}^{-1}$ and $3334 \mathrm{~cm}^{-1}\left(v_{\mathrm{s}}(\mathrm{N}-\mathrm{H})\right), 1682 \mathrm{~cm}^{-1}\left(v_{\mathrm{s}}(\mathrm{C}=\mathrm{O})\right), 1458 \mathrm{~cm}^{-1}\left(v_{\mathrm{s}}(\mathrm{CN})\right), 1589 \mathrm{~cm}^{-1}(\delta(\mathrm{N}-\mathrm{H}))$ and $1139 \mathrm{~cm}^{-1}$ and $1033\left(\mathrm{vas}_{\mathrm{as}}(\mathrm{CN})\right)$. Comparing urea spectrum with the spectrum of the assynthesized NPs and the NPs calcined at $300^{\circ} \mathrm{C}$ we observed four differences: 1) the peak at $1682 \mathrm{~cm}^{-1}$, related with $\mathrm{C}=\mathrm{O}$ symmetric stretching, is shifted to $1623 \mathrm{~cm}^{-1} ; 2$ ) the peak at $1589 \mathrm{~cm}^{-1}$, which was assigned to the deformation of the $\mathrm{NH}$ bond, is shifted to $1565 \mathrm{~cm}^{-1}$; 3) the peaks at $3431 \mathrm{~cm}^{-1}$ and $3334 \mathrm{~cm}^{-1}$, which correspond to the symmetric stretching of the primary amine, are broader and overlapped; and 4) the peak at $1387 \mathrm{~cm}^{-1}$, characteristic of nitrate $\left(\mathrm{NO}_{3}{ }^{-}\right)$, only appears in the spectrum of the assynthesized NPs and the NPs calcined at $300^{\circ} \mathrm{C}$. The former three differences suggest that the urea molecules are coordinated to the metal, $\mathrm{Zr}$ (IV) and /or Y (III), contained in crystal lattice of the YSZ-NPs. That result along with the appearance of the $\mathrm{NO}_{3}{ }^{-}$ characteristic peak points to the formation of a complex that can be written as $\left[(\mathrm{YSZ})\left(\mathrm{CON}_{2} \mathrm{H}_{2}\right)_{\mathrm{x}}\right]\left(\mathrm{NO}_{3}{ }^{-}\right)_{\mathrm{y}}$, where the urea molecules are the ligands and $\mathrm{NO}_{3}{ }^{-}$is the counter anion. The presence of those ligands was also confirmed by thermal analysis 
(Figure 6). The $\mathrm{Tg}$ analysis (Figure 6, red line) shows a loss of $24 \%$ of the total weight. The first weight loss $(\sim 5 \%)$ was observed at $100^{\circ} \mathrm{C}$ and is associated, among other phenomena, to desorption of adsorbed water. ${ }^{24}$ The $\left(\mathrm{NO}_{3}\right)^{-}$ions from the $\left[(\mathrm{YSZ})\left(\mathrm{CON}_{2} \mathrm{H}_{2}\right)_{\mathrm{x}}\right]\left(\mathrm{NO}_{3}{ }^{-}\right)_{\mathrm{y}}$, along with the ammonium anion $\left(\mathrm{NH}_{4}{ }^{+}\right)$that came from the hydrolysis of urea leaded to $\left(\mathrm{NH}_{4}\right) \mathrm{NO}_{3}$. The exothermic peak centered at $254^{\circ} \mathrm{C}$ depicted in the TGA (Figure 6, black line) is assigned to the sublimation and dissociation of this compound. ${ }^{25}$ Finally, the broad exothermic peak that appears at temperatures $>450^{\circ} \mathrm{C}$, can be related to different processes occurring in conjunction with the oxidation of the urea that was linked to the NPs ${ }^{26}$, such as the unreacted urea, the $\mathrm{ZrO}_{2}$ crystallization which came from the unreacted fraction of $\mathrm{ZrO}\left(\mathrm{NO}_{3}\right)_{2} \cdot \mathrm{xH}_{2} \mathrm{O}$ and phase transformations of the residual monoclinic $\mathrm{ZrO}_{2}$ observed by Raman ${ }^{27}$.

The urea linked to the YSZ NPs is acting as an intern dispersant since it came from the NPs synthesis. In order to confirm it, its ability to stabilize the NPs was studied by DLS. Figure 7 shows the distributions of the hydrodynamic sizes in volume of the assynthesized NPs (blue line) and the NPs after sonication for $2 \mathrm{~min}$. The hydrodynamic size of the as synthesized NPs, $\mathrm{d}(\mathrm{v} 50)_{\mathrm{as}}=1154 \pm 178 \mathrm{~nm}$ (table II) shifted down to $\mathrm{d}(\mathrm{v} 50)_{\mathrm{d}}=83 \pm 15 \mathrm{~nm}$ after $2 \mathrm{~min}$ of sonication. That result suggests that the agglomerates were broken down in smaller systems (urea-NPs) of aggregated NPs (this system cannot break down in smaller systems by physical methods) by the action of the ultrasounds energy. The aggregated NPs remained at that size due to the urea adsorbed on the NP surfaces prevents the re-agglomeration acting as a dispersant. The dispersant power of the urea adsorbed on the surface of the NPs can be improved exchanging it for a high molecular weight $(\mathrm{Mw}=25000)$ organic molecule like polyethyleneimine $(\mathrm{PEI})$ is. The urea adsorbed on the NP surfaces is firstly activated by adding $\mathrm{HNO}_{3}$ up to $\mathrm{pH}=5$ and PEI. Based on previous studies, 1 wt.\% of PEI was considered the optimum quantity for particle dispersion and stabilization ${ }^{15,21,28}$. The urea activated as a cation $\left(\left[\mathrm{COHN}_{2} \mathrm{H}_{4}\right]^{+}\right)$ facilitated the exchange with PEI by a substitution mechanism $\left(\mathrm{S}_{\mathrm{N}}\right)$. When the equilibrium was achieved (the $\mathrm{pH}$ was stabilized at 5) the suspension was sonicated for 2 min to break the agglomerated NPs in smaller systems. 20h later, the size of the new aggregates with PEI adsorbed on the NP surface remained at $\mathrm{d}(\mathrm{v} 50)_{\mathrm{s}}=74 \pm 16 \mathrm{~nm}$ (Figure 7, green line; table II). As we expected, due to the steric effect of PEI is larger than urea their ability to disperse is also higher.

The as-stabilized YSZ NPs were deposited onto the negatively charged and polished steel substrates by cathodic electrophoretic deposition (EPD). The as-stabilized YSZ 
NPs hold at $\mathrm{pH}=5$ positive surface charge with Zeta values $>50 \mathrm{mV}$ ), high electrophoretic mobility $\left(\mu=2.1 \pm 0.1 \times 10^{-4} \mathrm{~cm}^{2} \mathrm{~V}^{-1} \mathrm{~s}^{-1}\right)$, and also adequate conductivity $\left(\sigma=200 \pm 5 \mu \mathrm{S} \mathrm{cm}^{-1}\right)$ to move and be deposited by EPD. ${ }^{7,} 29$ Electropolished steel substrates were used to avoid anode interferences during both the deposition process and the films characterization. The EPD conditions were adjusted to maintain an electric field of $1.5 \mathrm{~V} \mathrm{~cm}^{-1}$ from 0 to $60 \mathrm{~min}$.

The morphology of the as-deposited film surfaces was scanned using a dynamic mode in order to preserve the properties of the non-sintered films. Figure 8a shows the histogram of the as-deposited films topography deposited during 15, 30 and $60 \mathrm{~min}$. Differences in the height distribution evidence slight differences among film surfaces during the coating growth. Those variations are related to the dissimilarities observed between the $2 \times 2 \mu \mathrm{m}$ topographies showed in Figures $8 \mathrm{~b}, 8 \mathrm{c}$ and $8 \mathrm{~d}$, which correspond to the surfaces of the films deposited during $15 \mathrm{~min}, 30 \mathrm{~min}$ and $60 \mathrm{~min}$, respectively. Table III summarizes the data of the roughness analysis. Both, the root mean square roughness $\left(\mathrm{RR}_{\mathrm{MS}}\right)$ and average height $\left(\mathrm{H}_{\mathrm{Av}}\right)$, increase slightly with the deposition time. The largest variation is observed for the film deposited during $60 \mathrm{~min}$. Otherwise, the number of hills/holes prevails in the topography of the $15 \mathrm{~min}$ film (Figure $8 \mathrm{~b}$ ) leading to a sharper structure than those obtained after 30 and $60 \mathrm{~min}$ of deposition (Figure $8 \mathrm{c}$ and $8 \mathrm{~d}$, respectively). Surface kurtosis roughness $\left(\mathrm{R}_{\mathrm{ku}}\right)$, is used to measure the distribution of the spikes above and below the mean line/plane. In general, for spiky surfaces, $R_{k u}>3$; for bumpy surfaces, $\mathrm{R}_{\mathrm{ku}}<3$; perfectly random surfaces have kurtosis 3 . For our films, $\mathrm{R}_{\mathrm{ku}}$ is slightly higher than 3 for films deposited during $15 \mathrm{~min}$ and $30 \mathrm{~min}$. Hence, we can consider these surfaces almost perfectly random surfaces, however, it is noticed that this value increases with time leading to a bumpy surface after 60 min of deposition. The topographies showed in Figure 8 verified that in the surface of the film obtained after $15 \mathrm{~min}$ of deposition, the particle arrangement is lower and spikier, becoming slightly taller but remarkably smooth during film growth as is observed in the topographies of the films deposited during 30min and 60min (Figure 8c and 8d, respectively). Therefore, differences in the surface morphology evidence that the longer the deposition time, the larger the size of the aggregates deposited, suggesting a stepped growth which should start with the arrangement of the smallest aggregates.

Figure 9 shows the Atomic Force Microscopy (AFM) topography of the as-prepared YSZ film after $60 \mathrm{~min}$ of deposition. Figure 9a shows a $5 \times 5 \mu \mathrm{m}$ image in which large 
arrays are disposed forming islands over a homogeneous sub-layer. Additionally, Figure $9 \mathrm{~b}$ shows the scanning of a 10 times smaller area $(0.5 \times 0.5 \mu \mathrm{m})$ of the sub-layer at Figure 9a, and Figure 9c depicts a detail of this sub-structure. The roughness analysis of the $5 \times 5 \mu \mathrm{m}$ topography indicates that the maximum height $\left(\mathrm{H}_{\max }\right)$ of $\sim 80 \mathrm{~nm}$ is within the same range that the mean hydrodynamic size of the as-stabilized aggregates $\left(\mathrm{d}(\mathrm{v} 50)_{\mathrm{s}}\right.$ $=74 \pm 16 \mathrm{~nm})$. Hence, we considered that the as-stabilized aggregates of the asdeposited film are building blocks of the film, while the surface arrangement indicates a Frank-van-der-Merwe growth. ${ }^{30}$ Those measurements are in the same order of magnitude that the roughness data indicated in table III for this sample (film deposited during $60 \mathrm{~min}) . \mathrm{H}_{\max }$ is $50 \mathrm{~nm}$, which is approximately, the half of the mean hydrodynamic size of the as-stabilized aggregates dispersed with US and stabilized with PEI $\left(\mathrm{d}(\mathrm{v} 50)_{\mathrm{s}}=74 \pm 16 \mathrm{~nm}\right)$ and corresponds to the sub-layer observed in Figure 9c and 9d.

The $0.5 \times 0.5 \mu \mathrm{m}$ image shows $\mathrm{H}_{\max }$ of $5 \mathrm{~nm}$ (figure 9c). In this topography, it is possible to recognize the YSZ NPs formed during the synthesis, which their size is close to the $7.3 \pm 1.5 \mathrm{~nm}$ diameter (table II) obtained from the HR-TEM images (Figure 3). The YSZ NPs exhibit a full hexagonal packing arrangement (Figure 9c), which is the most compact and the lowest energy packing for spherical shapes ${ }^{31}$. This configuration is the most favorable structure when NPs display differences in their surface (such as charge, molecules, etc.) suggesting that during the synthesis, YSZ nanoparticles $7.3 \pm$ $1.5 \mathrm{~nm}$ arranges forming spheres-like aggregates of $15 \pm 2 \mathrm{~nm}$ size, which turn to form stable aggregates of $\mathrm{d}(\mathrm{v} 50)_{\mathrm{s}}=74 \pm 16 \mathrm{~nm}$ at the end of the synthesis process. AFM images verify that those aggregates are the building blocks of the electric-driven assembly. Those results identified a layer-by-layer growth mechanism in these films, a similar behavior that other authors have evidenced for the EPD of ordered monolayers. 32,33

Figure 10 shows the SEM images of the as-deposited films. A general view of these films was provided in figure 10a. Macroscopically, in all cases a highly packed NPbased film covers the substrate. For low deposition times, that means for the thinnest films, coatings became transparent. As deposition time increases films turn out to be translucent. Surface inspection of the as-deposited $60 \mathrm{~min}$ film (figure 10b) verifies the homogeneous structure and the uniformity of the coating at microscale range. The nanostructure of the as-deposited films is observed in both micrographs (figure 10b and 
10c), and the layer-by-layer (Frank-van-der-Merwe) growth is confirmed. ${ }^{30}$ The existence of agglomerates of $85 \mathrm{~nm}$ in size composed by smaller entities or aggregates (figure 10b) is also proved. The sticky and dense packing of YSZ NPs displayed in the micrograph prove the relevance of re-arrangement and squeezing of deposited NPs under the application of an electric field. ${ }^{15}, 34-36$ Re-arrangement and subsequence densification of the film are provided by the presence of PEI and urea still adsorbed on the NP surfaces, but neutralized under the surrounding conditions at the cathode (step 3 at figure 1). ${ }^{15}$

Figure 11 shows the micrographs of the YSZ films deposited during $60 \mathrm{~min}$ and calcined at $500^{\circ} \mathrm{C}$. The micrograph in figure $11 \mathrm{a}$ corresponds to the bended area of the film as indicates the inset image, while figure $11 \mathrm{~b}$ and $11 \mathrm{c}$ correspond to images of a flat sample. In the light of the XRD, Raman and thermal analysis (Figure 3a, 3b and 6, respectively) organic modifiers (PEI and urea) as well as water were eliminated at temperatures lower than $500{ }^{\circ} \mathrm{C}$. SEM micrographs of the sintered film at $500^{\circ} \mathrm{C}$ (Figure 11) illustrates that the amount of organic modifiers, lower than 2 wt.\%., used for dispersion and electro-conduced assembly of the nanoparticles does not develop porous in the nanostructured coatings, as has been demonstrated elsewhere. ${ }^{37}$ Homogeneous and crack-free films were obtained after sintering the films at $500{ }^{\circ} \mathrm{C}$ in a regular thermal treatments. ${ }^{36,38}$ After sample bending (figure 11a), the YSZ coating fails as a cohesive material suggesting the existence of a consolidated structure after the thermal treatment at unusually low temperature $\left(500^{\circ} \mathrm{C}\right)$. Moreover, the cross section of the YSZ film showed in Figure 11a, evidences it is a thin coating. The micrographs of the flat sintered films (Figure11a and Figure11b) show the structural integrity of the finished ceramic coating covering the metal substrate. The micrographs showed in figures $11 \mathrm{~b}$ and $11 \mathrm{c}$ verify the continuity and compactness of the nanostructure underneath of a more developed one. Moreover, the subjacent nanostructure observed by AFM (Figure 9 ) is observed in figure 11c and pointed out with white arrows.

The mentioned compactness of the EPD coatings where tested by Nanoindentation on samples depositing during 15 and $60 \mathrm{~min}$, as well as the coating depositing during 60 minutes and then thermally treated at $500{ }^{\circ} \mathrm{C}$. For comparative purposes an electropolished steel substrate and a sintered stainless-YSZ compact were also tested. Table IV collects the values of hardness $(\mathrm{H})$ and elastic modulus $(\mathrm{E})$. 
It is observed that the presence of the ceramic phase on the top of the steel increased the hardness of the system (green coating-substrate) without any thermal treatment. This increment is much more significant after the thermal treatment at $500^{\circ} \mathrm{C}$. Conversely, the elastic modulus did not increase maintaining values similar to the stainless-YSZ compact, whose characterization has been described elsewhere. ${ }^{39}$ The $\mathrm{H}$ values measured for the green coatings are similar to those obtained by pulsed plasma deposition ${ }^{40}$ indicating that the electric field conducted the particles to the substrate to form a very dense coating. It is known that electric field assisted the sintering of YSZ nanoparticles at $390^{\circ} \mathrm{C} .{ }^{41}$ In the process here presented, the high values obtained for the green coating suggests that the electric field facilitates the partial densification of the nanoparticles at room temperature.

The reliable and eco-friendly processing of such a kind of nanoparticle-based coatings has an unusual relevance in the nanoionics ${ }^{42}$, hence the electric and ionic conductivities linked with both, thickness and nanostructure of YSZ dense and crack free coatings is now under study.

The optical analysis, which is illustrated in Table $\mathrm{V}$ and described in detail in the supplementary information, indicates that the eco-one pot process proposed in this work leads to consolidated thin films $\left(36 \mathrm{~nm}\right.$ thickness after sintering at $\left.500^{\circ} \mathrm{C}\right)$ with high refractive index $(n=1.85 \pm 0.05)$ using temperatures lower than $500^{\circ} \mathrm{C}$.

The high values measured for the linear absorption coefficients $\alpha$ of the YSZ films (Figure 13, please see the supplementary information) which are the order of $10^{5}-10^{6}$ $\mathrm{cm}^{-1}$, evidence the high cristallinity of the deposited films. Moreover, the low $\mathrm{E}_{\mathrm{g}}$ determined for the YSZ films (Table IV) can be attribuited to a structural disorder induced by both the presence of $\mathrm{Y}^{3+}$ and the absence of $\mathrm{O}^{2-}$. The anion vacancies detected are the primary species in both solid-state conduction and heterogeneous electrocatalysis of solid oxide electrolytes, hence, the films developed in this work by the eco-one pot method would be suitable for the mentioned applications.

\section{Conclusions}

In summary, the viability of a one-pot approach that involves the electrophoretic deposition of YSZ NPs hydrothermally synthesized in mild conditions has been proven 
as a useful and eco-friendly tool for nanotechnology. A carefully selection of precursors (nitrates), synthesis modifier (urea) and dispersant (PEI) provided the optimum conditions for the consolidation of finished NP-based films benefiting from the synergy of the hydrothermal synthesis and the EPD, avoiding intermediated steps such as dry, mille, sieve or centrifuge.

The layer-by-layer or Frank-van-der-Merwe growth of an extremely densely packed NP-based film consolidated by green technologies was highlighted. Advantages of the proposed one-pot process, such as the high yield and reliability of the electrically driven assembly, have been validated.

Specifically for the YSZ nanostructure, a thin layer with structural integrity after calcination at an unusually low temperature as $500^{\circ} \mathrm{C}$ has been consolidated for the first time through a conventional NP processing route involved in the one-pot method. The film topography correlated with the as-stabilized NPs established that the electric-driven assembly of the stabilized NPs is crucial to achieve the extremely dense packing of the NPs. Moreover, the analysis of AFM micrographs indicated that the aggregated asstabilized NPs, which their diameter is $\sim 85 \mathrm{~nm},\left(\mathrm{~d}_{\mathrm{as}}(\mathrm{v} 50)\right)$ acted as building blocks in the shaping step. The building blocks were accurately inspected by AFM determining that their average size is $15 \pm 2 \mathrm{~nm}$ and they are constituted by YSZ NPs of $6.5 \pm 1.5$ $\mathrm{nm}$. Optimization of the $\mathrm{pH}$ and the concentration of surface modifiers facilitated rearrangement of the NPs improving the film density. Height gradient and surface morphology have been observed when deposition time increases. Differences in the topography of the film studied when the deposition time increased evidenced that the longer the deposition time, the larger the size of the aggregates deposited, suggesting a Frank van der Merwe growth which should start with the arrangement of the smallest aggregates. Hence, deposition time is the parameter directly related with important properties of the film like morphology and NP packing. Optical and mechanical analysis of both the green and the thermally treated coatings indicated that the as-dispersed NPs deposited by EPD leads to thin films with high refractive indexes and hardness values.

\section{Acknowledgments}


This work has been supported by the Spanish Ministry of Economy and competitiveness (MINECO) under contracts MAT 2009-14448-C02-01 and MAT2012 38650-C02-02. J.

A. Escribano acknowledges the Spanish Ministry of Science and Innovation for the concession of a FPI grant. 


\section{References}

1. Z. Xue, W. Zhang, X. Yin, Y. Cheng, L. Wang and B. Liu, RSC Advances, 2012, 2, 70747080.

2. G. Cao and D. Liu, Advances in Colloid and Interface Science, 2008, 136, 45-64.

3. I. Corni, M. P. Ryan and A. R. Boccaccini, Journal of the European Ceramic Society, 2008, 28, 1353-1367.

4. K. Zdansky and J. H. Dickerson, Sensors and Actuators, B: Chemical, 2013, 184, 295300.

5. D.-H. Ha, M. A. Islam and R. D. Robinson, Nano Letters, 2012, 12, 5122-5130.

6. L. Besra and M. Liu, Progress in Materials Science, 2007, 52, 1-61.

7. B. Ferrari and R. Moreno, Journal of the European Ceramic Society, 2010, 30, 10691078.

8. M. Ammam, RSC Advances, 2012, 2, 7633-7646.

9. A. Chávez-Valdez and A. R. Boccaccini, Electrochimica Acta, 2012, 65, 70-89.

10. J. A. Lewis, Journal of the American Ceramic Society, 2000, 83, 2341-2359.

11. W. M. Sigmund, N. S. Bell and L. BergstrÃ $₫ m$, Journal of the American Ceramic Society, 2000, 83, 1557-1574.

12. I. Gonzalo de Juan, A. Krejci and J. H. Dickerson, Langmuir, 2012, 28, 5295.

13. Alex J. Krejci, I. Gonzalo-Juan and J. H. Dickerson, ACS Applied Materials \& Interfaces, 2011, 3611

14. L. Besra, T. Uchikoshi, T. S. Suzuki and Y. Sakka, Journal of the European Ceramic Society, 30, 1187-1193.

15. M. Verde, M. Peiteado, A. C. Caballero, M. Villegas and B. Ferrari, Journal of Colloid and Interface Science, 2012, 373, 27-33.

16. A. J. Krejci, J. Mandal and J. H. Dickerson, Applied Physics Letters, 2012, 101, art. no. 043117.

17. I. Gonzalo-Juan, A. J. Krejci and J. H. Dickerson, in Key Engineering Materials, 2012, pp. 89-93.

18. Y. Castro, A. Duran, R. Moreno and B. Ferrari, Advanced Materials, 2002, 14, 505-508.

19. Y. Castro, B. Ferrari, R. Moreno and A. Duran, Surface \& Coatings Technology, 2004, 182, 199-203.

20. I. Gonzalo-Juan, B. Ferrari and M. T. Colomer, Journal of the European Ceramic Society, 2009, 29, 3185-3195.

21. I. Gonzalo-Juan, Thesis, 2010.

22. C. D. Wagner, L. E. Davis, M. V. Zeller, J. A. Taylor, R. H. Raymond and L. H. Gale, Surface and Interface Analysis, 1981, 3, 211-225.

23. A. E. Hughes and B. A. Sexton, Journal of Materials Science, 1989, 24, 1057-1061.

24. C. Tallon, R. Moreno and M. I. Nieto, International Journal of Applied Ceramic Technology, 2009, 6, 324-334.

25. M. Olszak-Humienik, Thermochimica Acta, 2001, 378, 107-112.

26. L. Muresan, E.-J. Popovici, R. Grecu and L. B. Tudoran, Journal of Alloys and Compounds, 2009, 471, 421-427.

27. M.-H. Lee, H.-Y. Lin and J. L. Thomas, Journal of the American Ceramic Society, 2006, 89, 3624-3630.

28. M. Verde, A. C. Caballero, Y. Iglesias, M. Villegas and B. Ferrariz, Journal of the Electrochemical Society, 2010, 157, H55-H59.

29. B. Ferrari and R. Moreno, Journal of the European Ceramic Society, 1997, 17, 549-556. 
30. E. Bauer, Zeitschrift für Kristallographie, 1958, 110, 372-394.

31. S. Jiang, Q. Chen, M. Tripathy, E. Luijten, K. S. Schweizer and S. Granick, Advanced Materials, 2010, 22, 1060-1071.

32. A. J. Krejci, C. G. W. Thomas, J. Mandal, I. Gonzalo-Juan, W. He, R. L. Stillwell, J.-H. Park, D. Prasai, V. Volkov, K. I. Bolotin and J. H. Dickerson, Journal of Physical Chemistry B, 2013, 117, 1664-1669.

33. I. Gonzalo-Juan, A. J. Krejci and J. H. Dickerson, Langmuir, 2012, 28, 5295-5301.

34. J. Dupta and H. Hofmann, Self-Organization of Colloidal Nanoparticles. Encyclopedia of Nanosience and Nanotechnology, American Scientific Publishers, 2000.

35. C. Z. Ji, W. H. Lan and P. Xiao, Journal of the American Ceramic Society, 2008, 91, 11021109.

36. J. A. Downs and V. M. Sglavo, Journal of the American Ceramic Society, 2013.

37. J. A. Lewis, J. Am. Cerm. Soc., 2000, 83, 2341-2354.

38. D. Hotza, A. Leo, J. Sunarso and J. C. D. Da Costa, Journal of Nano Research, 2009, 6, 115-122.

39. I. Gonzalo-Juan, P. Parente, A. J. Sanchez-Herencia, F. Rubio, R. Pareja and B. Ferrari, Solid State lonics, 2011, 190, 67-74.

40. M. Bianchi, A. Russo, N. Lopomo, M. Boi, M. C. Maltarello, S. Sprio, M. Baracchi and M. Marcacci, Journal of Materials Chemistry B, 2013, 1, 310-318.

41. J. A. Downs and V. M. Sglavo, Journal of the American Ceramic Society, 2013, 96, 13421344.

42. J. Maier, Nature Materials, 2005, 4, 805-815. 
Table I. Summary of the nanoparticle characterization after drying at $60^{\circ} \mathrm{C} / 24 \mathrm{~h}$ : density $(\rho)$, specific surface area $\left(\mathrm{S}_{\mathrm{s}}\right)$, average diameter obtained from TEM analysis $\left(\mathrm{d}_{\mathrm{TEM}}\right)$, and binding energies $(\mathrm{BE})$ of the oxygen $\mathrm{O}(1 \mathrm{~s})$ and of $\mathrm{Y}(3 \mathrm{~d}) / \mathrm{Zr}(3 \mathrm{~d})$ atomic ratio. The numbers in parenthesis next to the binding energies corresponds to the peak area percentages.

\begin{tabular}{|c|c|c|c|c|c|}
\hline & $\begin{array}{c}\rho^{*} \\
\left(\mathrm{~g} / \mathrm{cm}^{3}\right)\end{array}$ & $\begin{array}{c}\mathrm{S}_{\mathrm{s}}^{*} \\
\left(\mathrm{~m}^{2} / \mathrm{g}\right)\end{array}$ & $\begin{array}{l}\mathbf{d}_{\mathrm{TEM}}^{*} \\
(\mathrm{~nm})\end{array}$ & $\begin{array}{l}01 s^{*} \\
(\mathrm{eV})\end{array}$ & $\begin{array}{l}\mathrm{Y} / \mathrm{Zr} * \\
\text { (at) }\end{array}$ \\
\hline $\begin{array}{l}\text { YSZ nanoparticles } \\
\text { dried at } 60^{\circ} \mathrm{C} / 24 \mathrm{~h}\end{array}$ & 3.43 & 140 & 7.28 & $\begin{array}{l}530.0(68) \\
531.7(32)\end{array}$ & 0.174 \\
\hline
\end{tabular}

Experimental and calculated errors: $\rho \pm 10 \%, \mathrm{~S}_{\mathrm{s}} \pm 5 \%, \mathrm{~d}_{\mathrm{TEM}}( \pm 1.47 \mathrm{~nm}), \mathrm{BE}( \pm 0.1 \mathrm{eV})$

Table II: Schematic representation of the sizes of the different NP clustering along the one pot process: average diameter of an isolated NP obtained from TEM analysis $\left(\mathrm{d}_{\mathrm{TEM}}\right)$; an average diameter calculated using the absorption model from the nitrogen absorption isotherm $\left(\mathrm{d}_{\mathrm{BET}}\right)$; hydrodynamic size of the as synthesized NPs (d(v50)as) and hydrodynamic size of the NPs dispersed by US and stabilized with 1wt.\% of PEI $\left(\mathrm{d}(\mathrm{v} 50)_{\mathrm{s}}\right)$. The urea and PEI molecules adsorbed on NP surfaces are depicted as short and long positive chains, respectively.

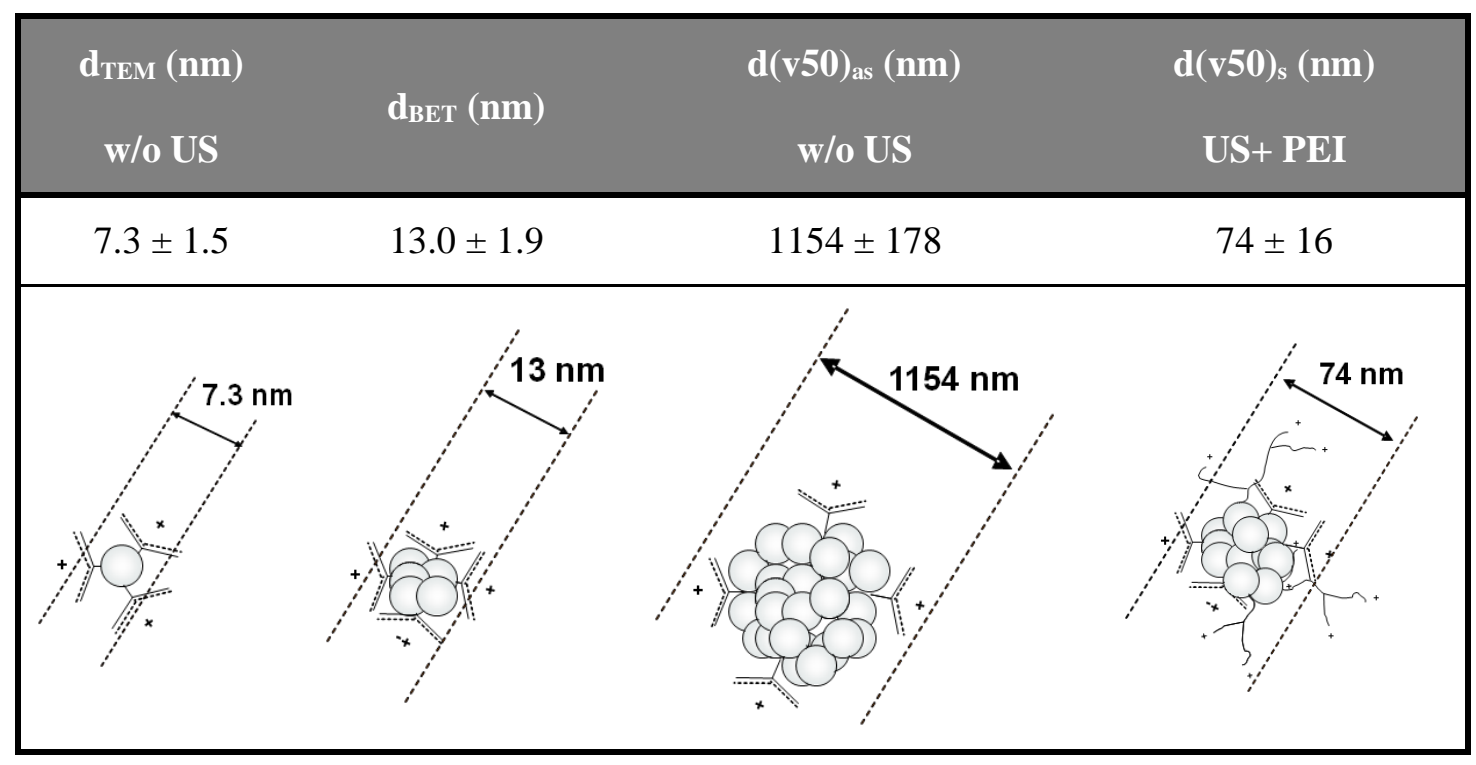

Table III. Summary of the roughness values of 15, 30 and 60 min as-deposited films: Root mean squared roughness, $\mathrm{RR}_{\mathrm{MS}}$, kurtosis roughness, $\mathrm{R}_{\mathrm{ku}}$, average height, $\mathrm{H}_{\mathrm{Av}}$, and maximum height, $\mathrm{H}_{\max }$.

\begin{tabular}{|ccccc|}
\hline Deposition Time & $\mathbf{R}_{\mathrm{RMS}}(\mathbf{n m})$ & $\mathbf{R}_{\mathrm{ku}}(\mathbf{n m})$ & $\mathbf{H}_{\mathrm{Av}}(\mathbf{n m})$ & $\mathbf{H}_{\mathrm{max}}(\mathbf{n m})$ \\
\hline $\mathbf{1 5} \mathbf{~ m i n}$ & 3.4 & 4.7 & 15 & 36 \\
\hline $\mathbf{3 0} \mathbf{~}$ in & 3.5 & 6.1 & 16 & 35 \\
\hline $\mathbf{6 0} \mathbf{~ m i n}$ & 4.9 & 7.7 & 19 & 50 \\
\hline
\end{tabular}


Table IV. Summary of the optical ( refractive index at $700 \mathrm{~nm}(\mathrm{n} 700 \mathrm{~nm})$ and energy band gap (Eg)) and mechanical values (hardness $(\mathrm{H})$ and elastic modulus $(\mathrm{E})$ ) of the 15 and 60 min as-deposited films (15 min-green and $60 \mathrm{~min}$-green, respectively) and of the $60 \mathrm{~min}$ deposited film annealed at $500^{\circ} \mathrm{C}(60 \mathrm{~min}$ sintered at $\left.500^{\circ} \mathrm{C}\right)$. The mechanical properties of a stainless-YSZ compact and an electropolished stainless (ALSI 304) are also included.

\begin{tabular}{|cccccc|}
\hline Coating & $\begin{array}{c}\mathrm{d} \\
(\mathrm{nm})\end{array}$ & $\begin{array}{c}\mathrm{E}_{\mathrm{g}} \\
(\mathrm{eV})\end{array}$ & $\begin{array}{c}\mathrm{H} \\
(\mathrm{GPa})\end{array}$ & $\begin{array}{c}\mathrm{E} \\
(\mathrm{GPa})\end{array}$ \\
\hline $\mathbf{1 5}$ min-green & $14.0 \pm 0.1$ & $1.03 \pm 0.05$ & $2.97 \pm 0.03$ & $5.2 \pm 0.9$ & $151 \pm 17$ \\
\hline $\begin{array}{c}\mathbf{6 0} \text { min-green } \\
\text { 60 min-sintered } \\
\text { at 500 } \mathbf{C}\end{array}$ & $53.0 \pm 0.1$ & $1.13 \pm 0.05$ & $3.49 \pm 0.03$ & $4.3 \pm 0.5$ & $135 \pm 30$ \\
\hline $\begin{array}{c}\text { Stainless-YSZ } \\
\text { compact }\end{array}$ & - & $1.85 \pm 0.05$ & $2.69 \pm 0.03$ & $6.9 \pm 2.0$ & $145 \pm 10$ \\
\hline ALSI 304 & - & - & - & $12.0 \pm 0.2$ & $131 \pm 24$ \\
\hline
\end{tabular}




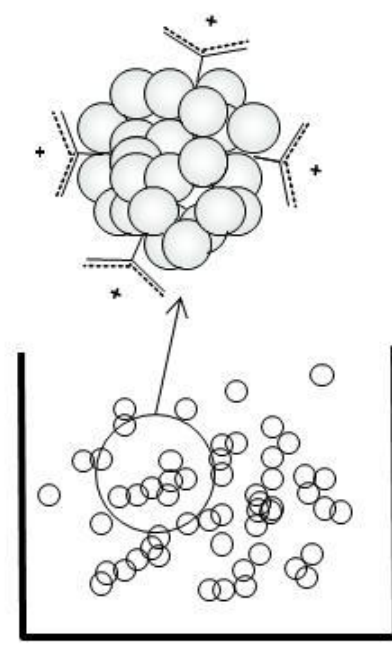

1. Hydrothermal Synthesis

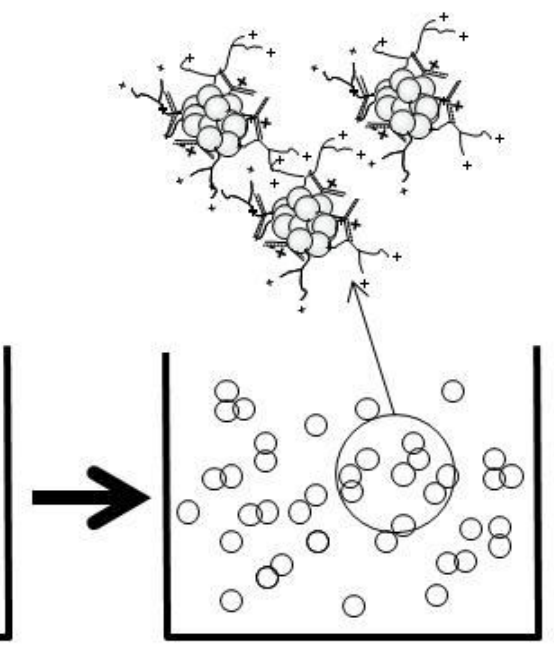

2. Dispersion + Stabilization
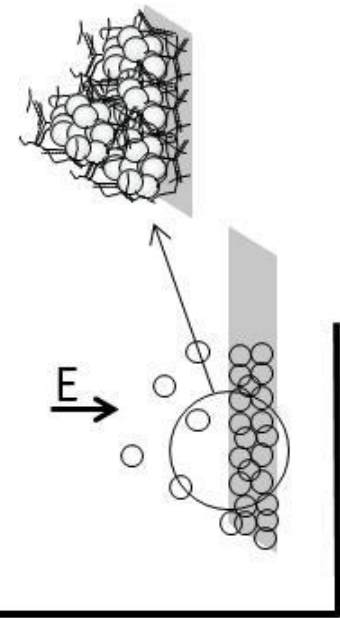

3. Electrophoretic Deposition

Figure 1. Scheme of the one pot process including the configuration of the NPs aggregation at different steps of the process: hydrothermal synthesis (1), dispersion by ultrasound and nanoparticle stabilization with PEI (2) and electrophoretic deposition (3). In the upper draw single particles correspond to synthesized NPs, short and long positive chains correspond to urea and PEI adsorbed on the NP surfaces, respectively.

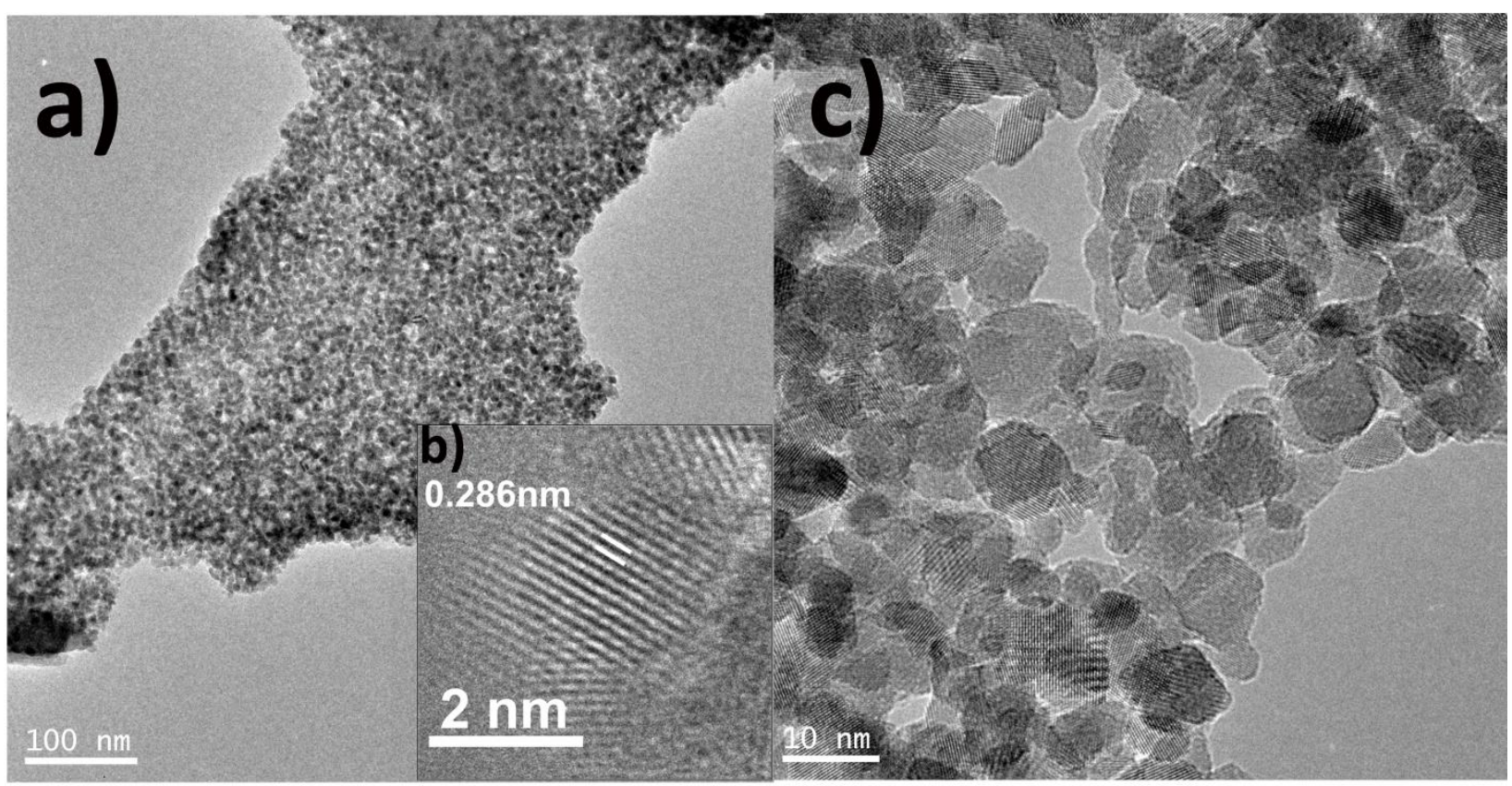

Figure 2. TEM micrographs (a, b, c) of the as-synthesized YSZ nanoparticles using 1:3 urea: $\left(\mathrm{ZrO}_{2}\right)$ ratio. The primary diameter of the sphere-like nanocrystals is $7.28 \pm 1.47 \mathrm{~nm}$. The lattice fringes (b) indexed is $0.286 \pm 0.01 \mathrm{~nm}$ which corresponds to the 111 plane of 8YSZ (ASTM 30-1468). 

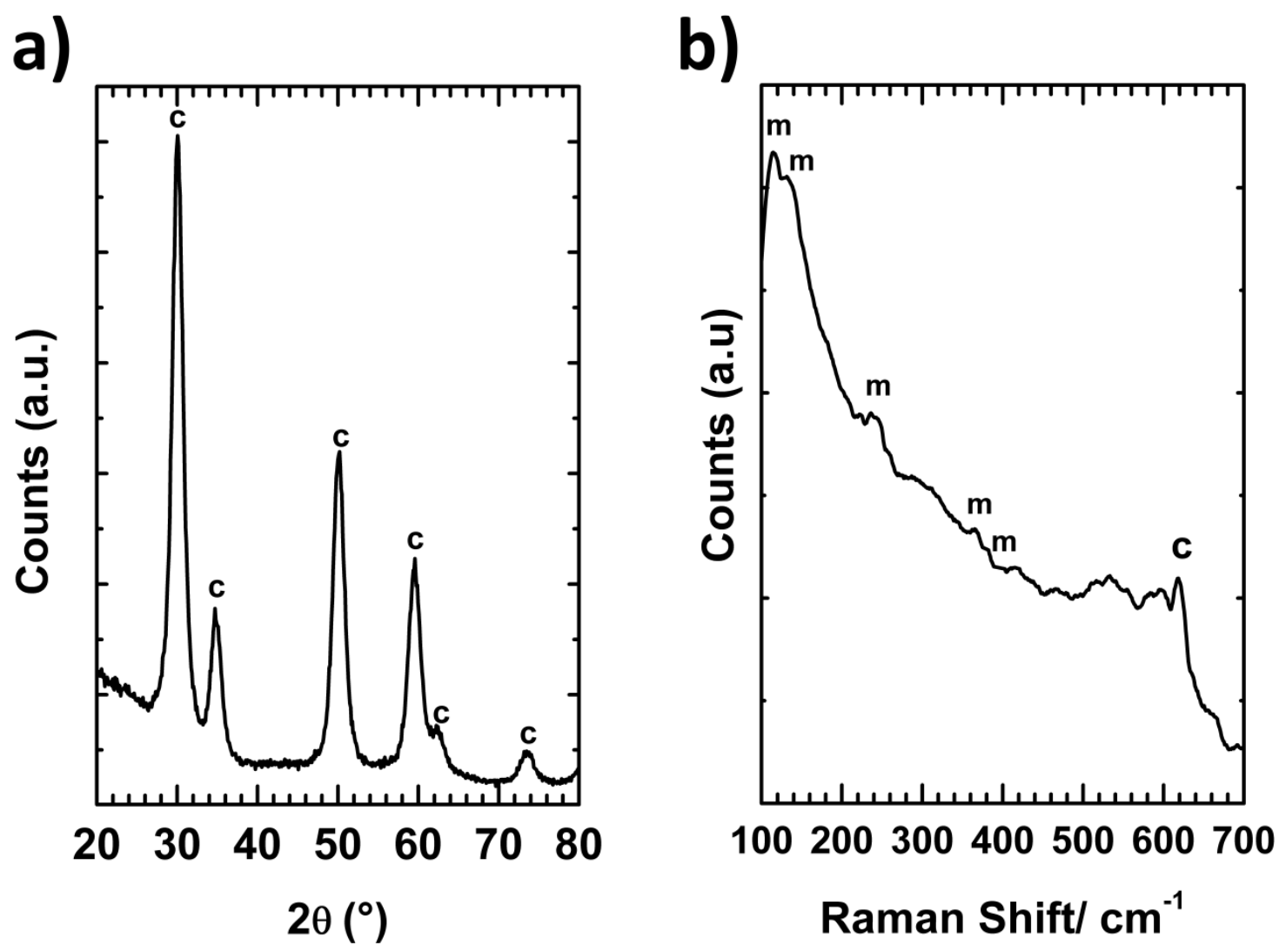

Figure 3. XRD (a) and Raman (b) spectra of the as-synthesized YSZ nanocrystals after drying at $60^{\circ} \mathrm{C}$. The symbol $\mathbf{c}$ showed in the XRD spectrum correspond to cubic phase according to the reference diffraction peak locations of 8YSZ (ASTM 30-1468). The $\mathbf{m}$ and $\mathbf{c}$ symbols depicted on Raman spectrum correspond to monoclinic and cubic phase, respectively.

a)

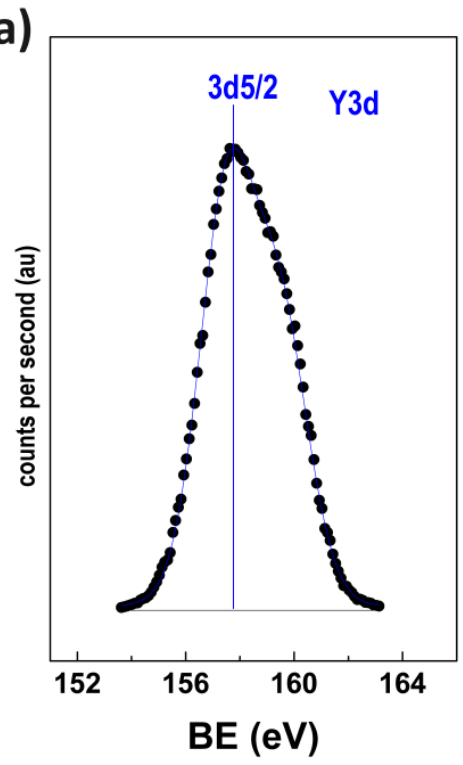

b)

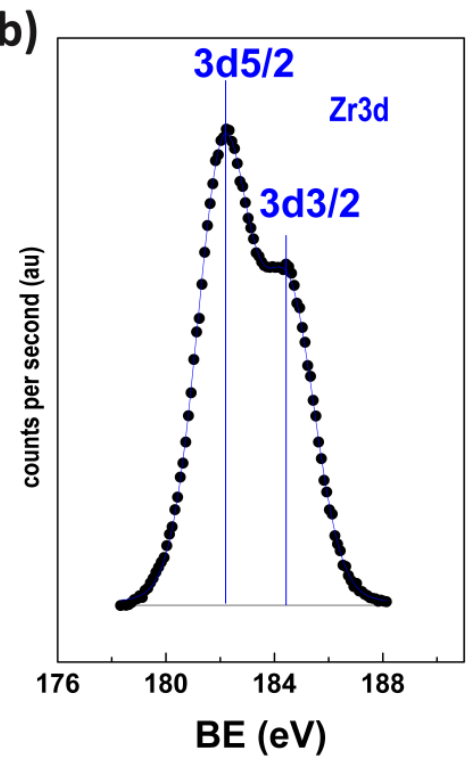

c)

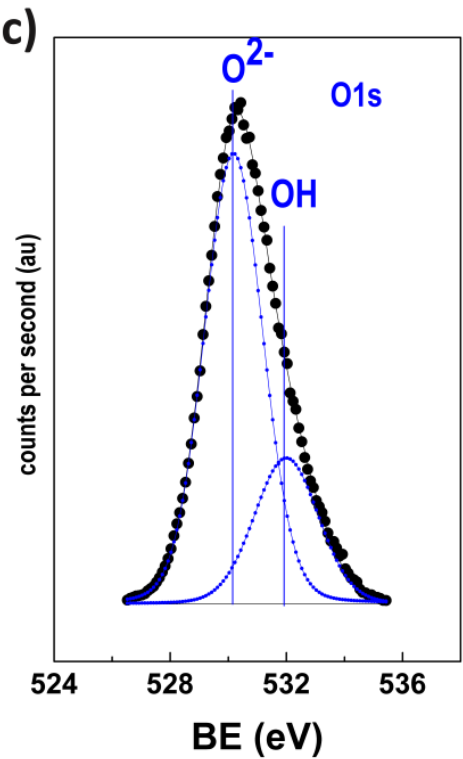

Figure 4. XPS spectra: $\mathrm{Y} 3 \mathrm{~d}(\mathrm{a}), \mathrm{Zr} 3 \mathrm{~d}$ (b) and $\mathrm{O}$ 1s (c) of as-synthesized YSZ nanoparticles dried at $60^{\circ} \mathrm{C}$ during $24 \mathrm{~h}$. 


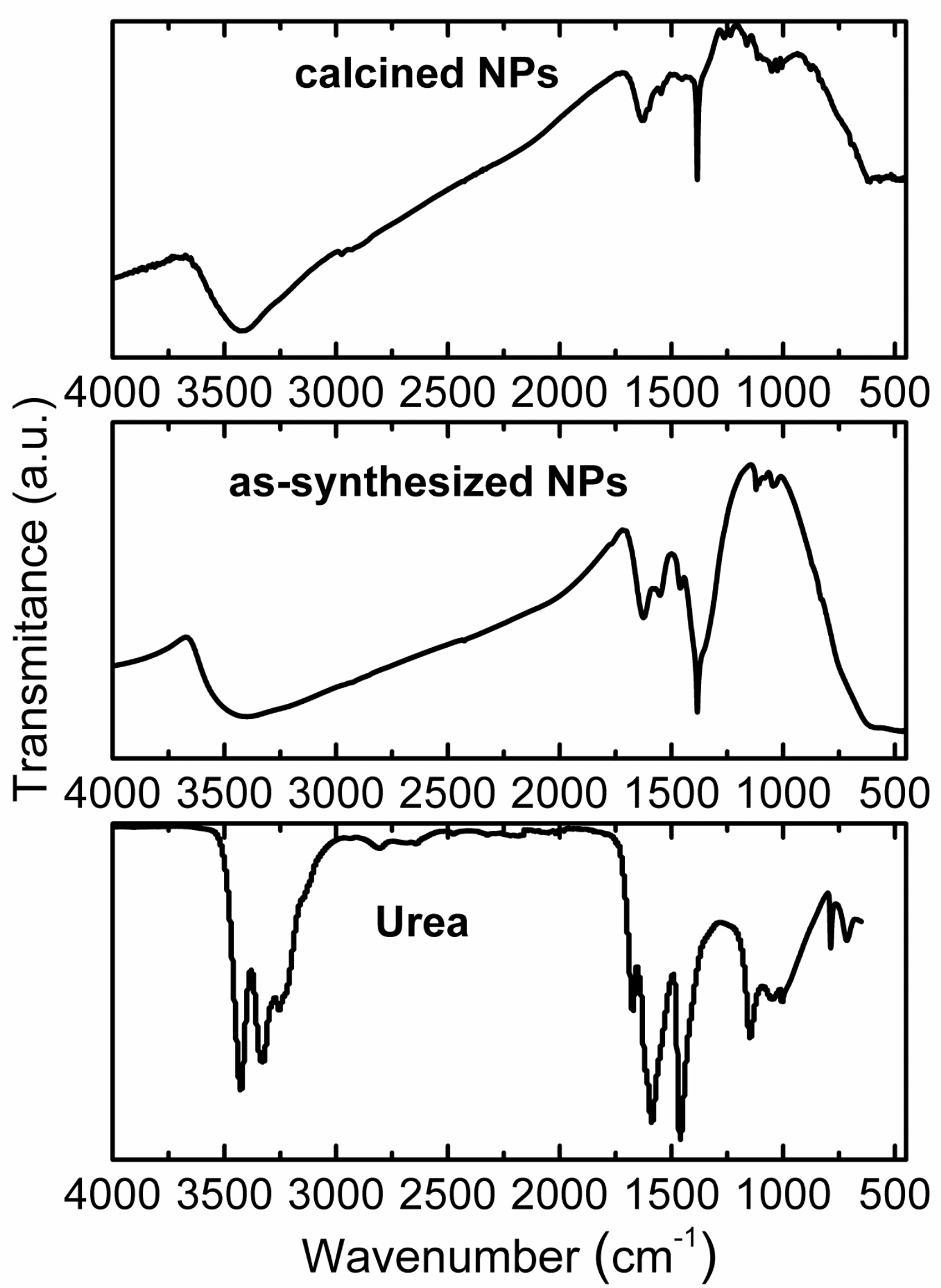

Figure 5. FTIR spectra of the urea, as-synthesized NPs and the NPs calcined at $300^{\circ} \mathrm{C} /$ $30 \mathrm{~min}$. 


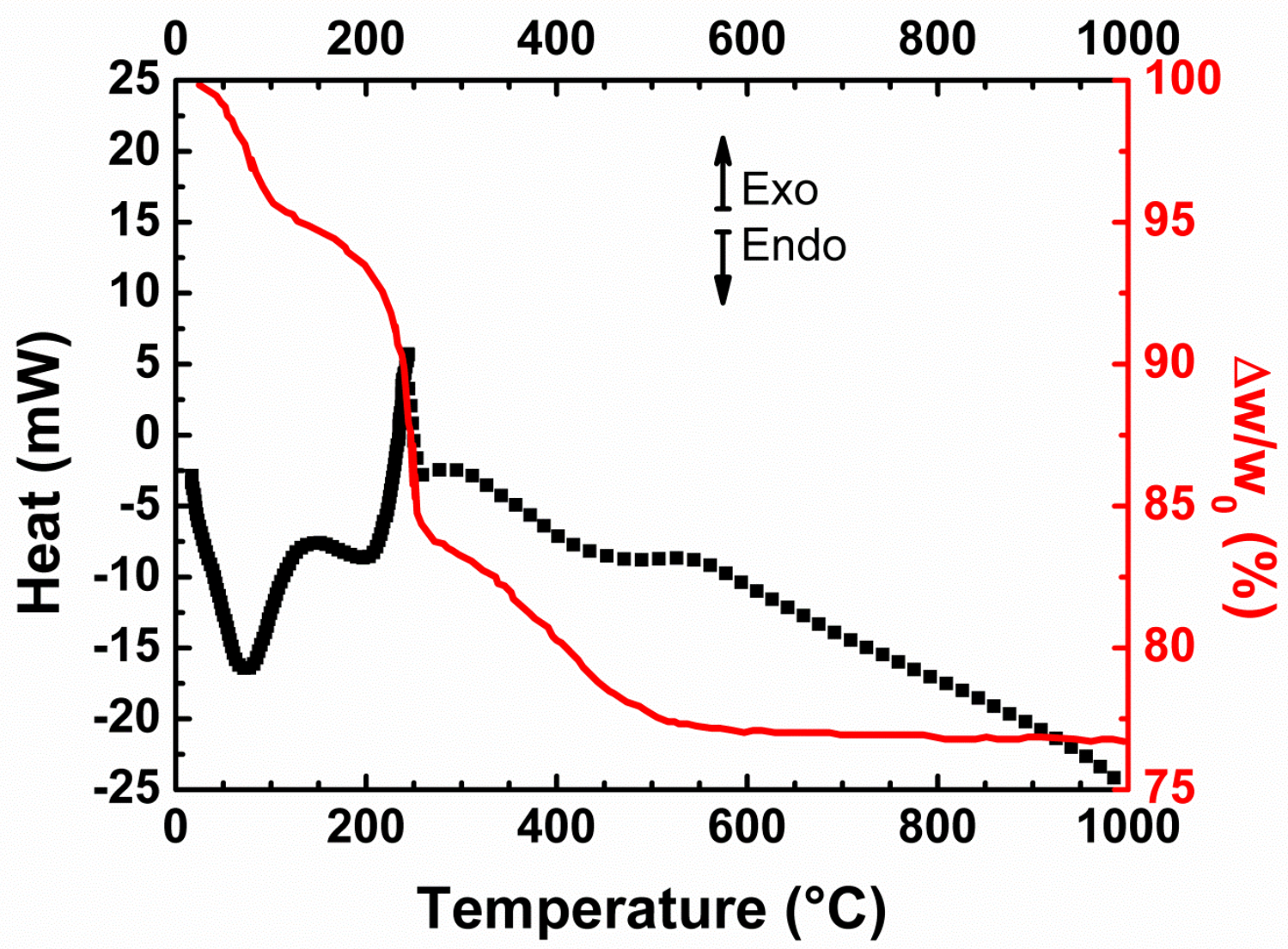

Figure 6. Thermal analysis of the of as-synthesized YSZ nanoparticles.

Thermogravimetric analysis (TGA) corresponds to the black line and the differential thermal analysis (DTA) corresponds to the red line. 


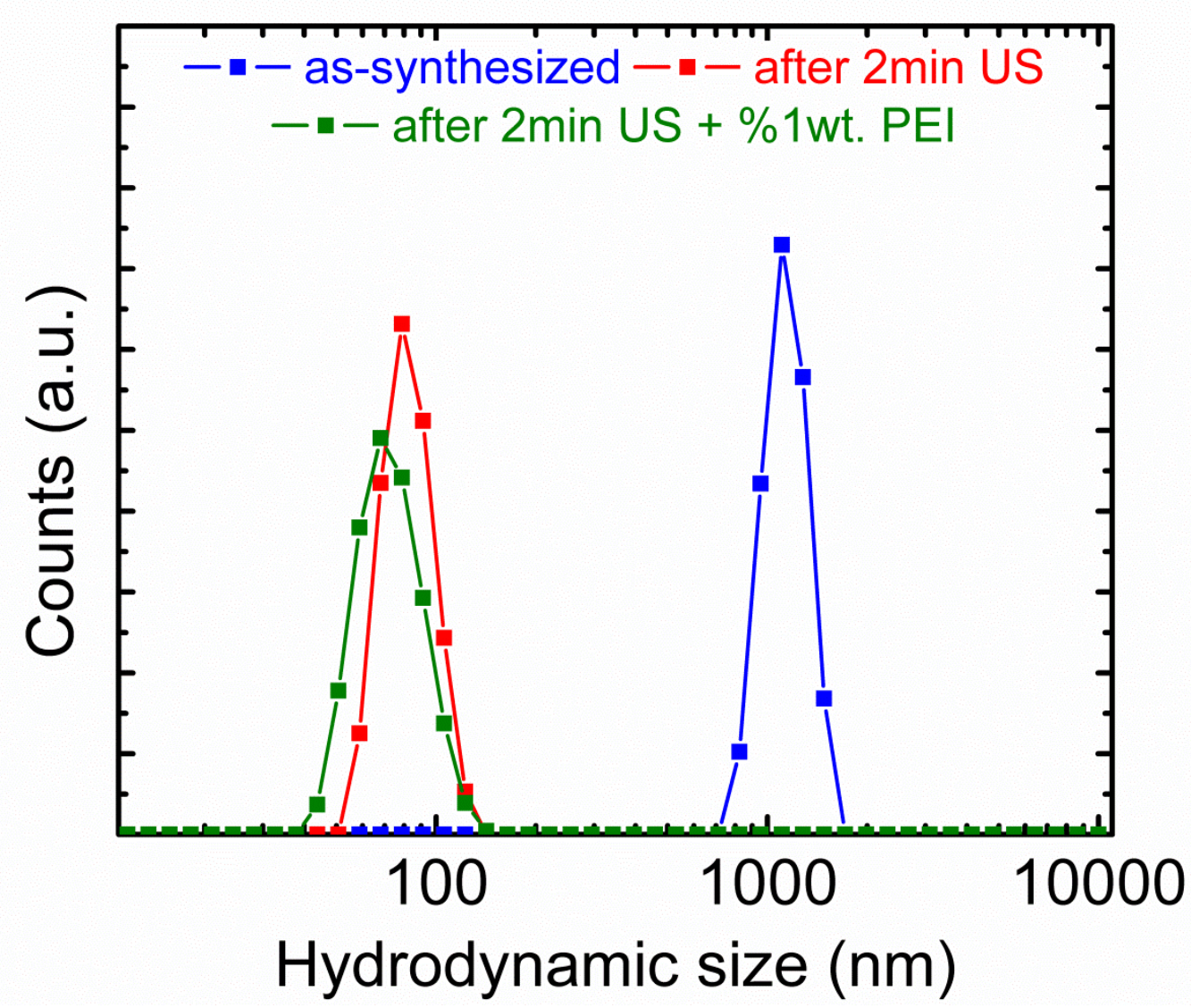

Figure 7. Hydrodynamic diameter distributions of YSZ nanocrystals suspended in the synthesis mother water as a function of their dispersion: as synthesized (blue line), after sonication during $2 \mathrm{~min}$. (red line) and after sonication during $2 \mathrm{~min}$ following by nanoparticle stabilization with $1 \%$ PEI (green line). The average diameter for the assynthesized nanoparticles is $\mathrm{d}(\mathrm{v} 50)_{\mathrm{as}}=1154 \pm 178 \mathrm{~nm}$ which shifted down to $\mathrm{d}(\mathrm{v} 50)_{\mathrm{d}}=83 \pm 15 \mathrm{~nm}$ after $2 \mathrm{~min}$ of sonication and remained $\mathrm{d}(\mathrm{v} 50)_{\mathrm{s}}=74 \pm 16 \mathrm{~nm} 20 \mathrm{~h}$ later than the nanoparticles were sonicated and stabilized with $1 \mathrm{wt} . \%$ PEI. 


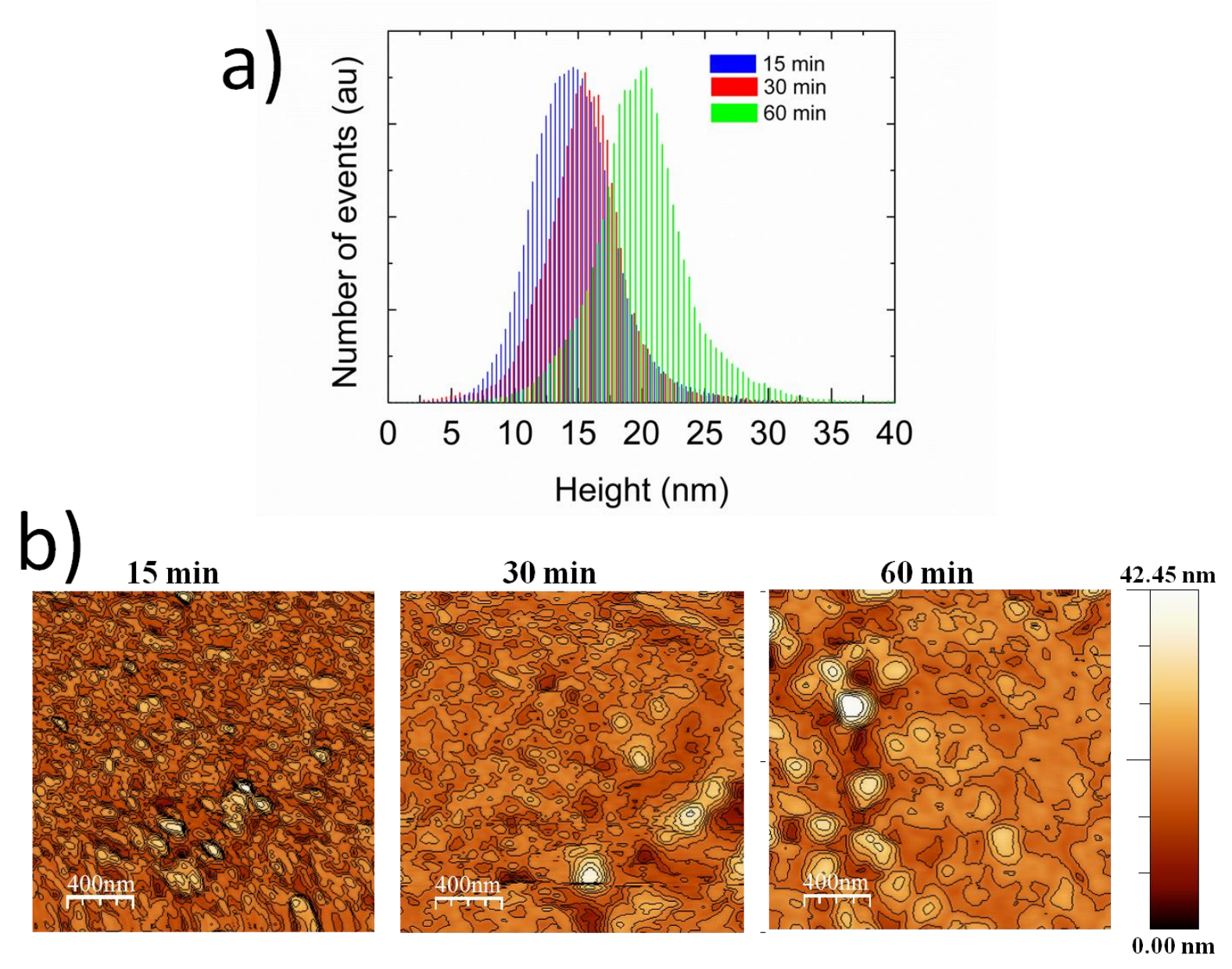

Figure 8. Surface height distributions (a) and AFM topographies of the films electrophoretically deposited after (b) $15 \mathrm{~min}$, (c) $30 \mathrm{~min}$ and (d) $60 \mathrm{~min}$.

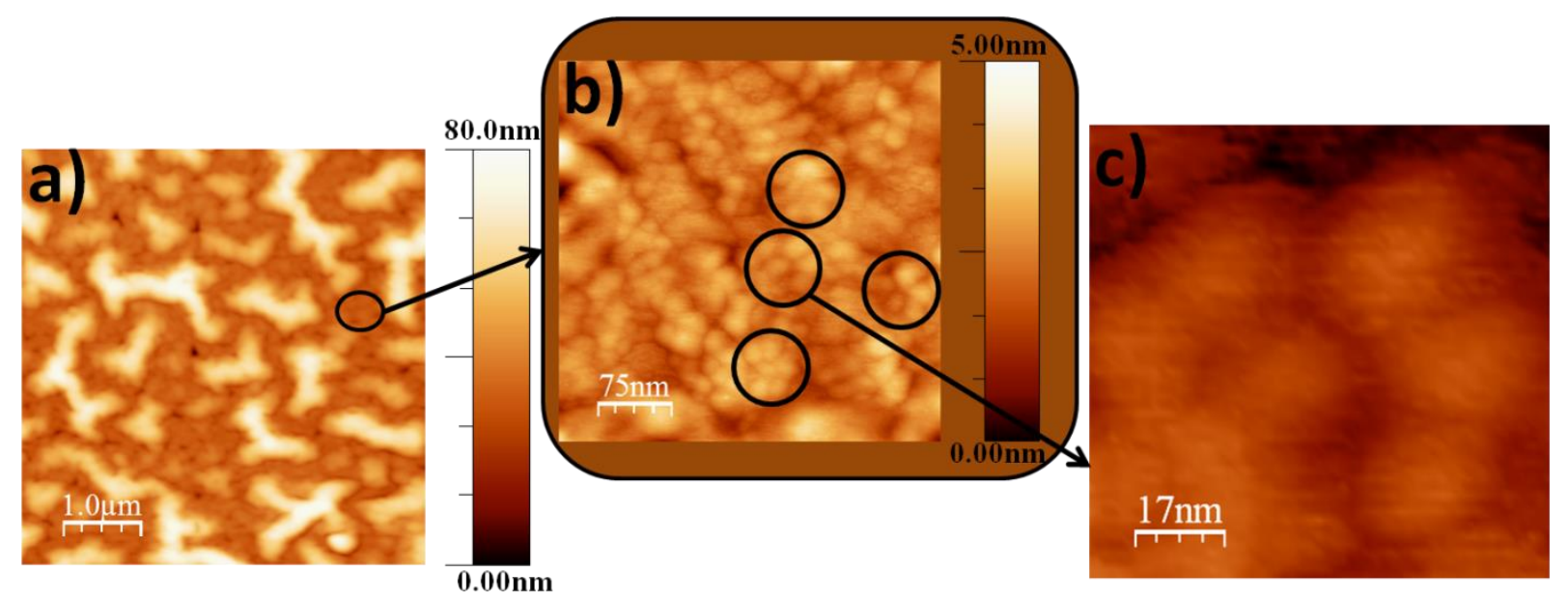

Figure 9. AFM images of the YSZ film electrophoretically deposited applying $1.5 \mathrm{~V}$ $\mathrm{cm}^{-1}$ during 60min at different magnifications: $5 \times 5 \mu \mathrm{m}$ area of the film (a), $0.5 \times 0.5 \mu \mathrm{m}$ 
area of the 60 min in which the hexagonal packed nanocrystals, that acted as building blocks during the electrophoretic deposition, are pointed out by circles (b), and detailed by a higher magnification AFM image (c).

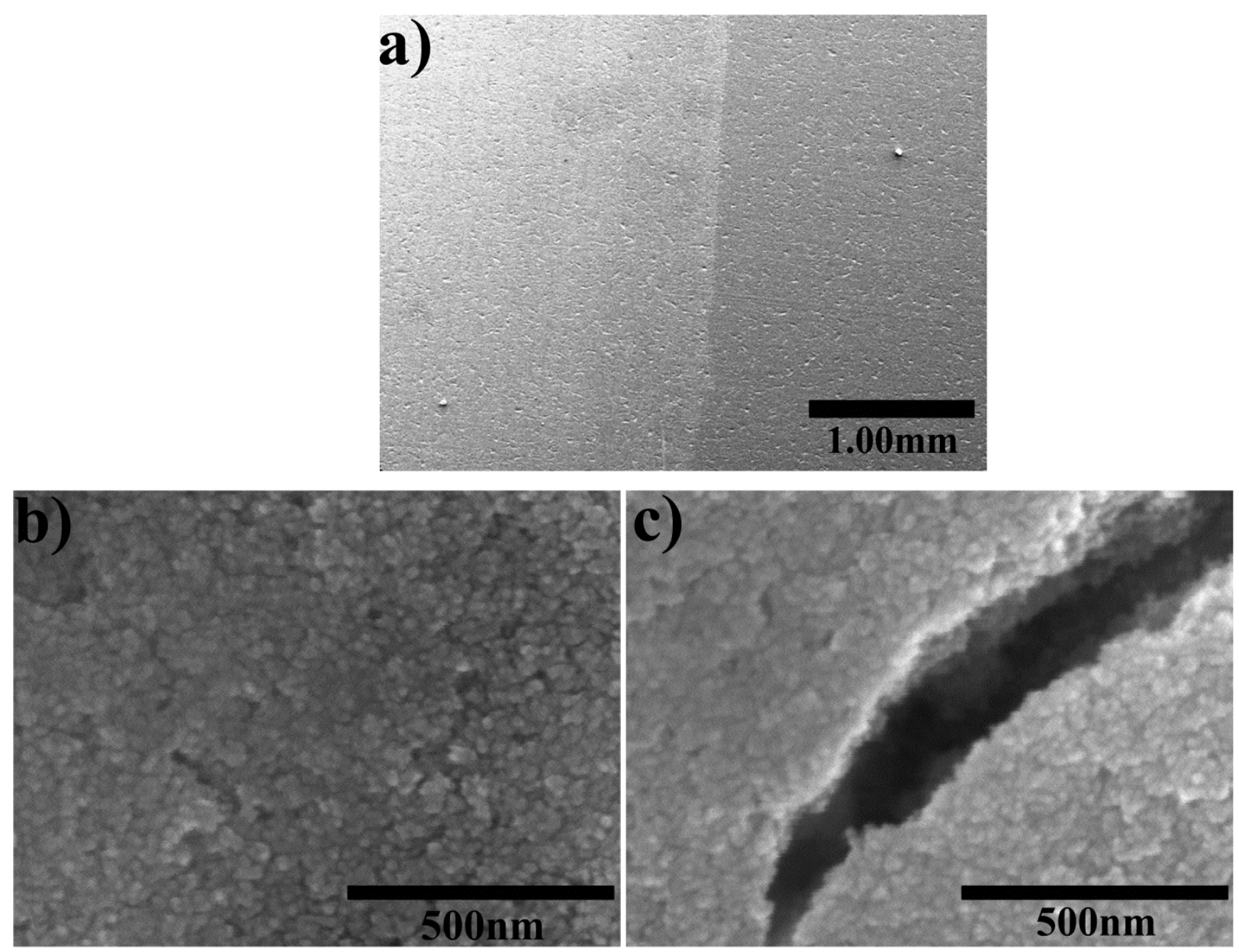

Figure 10. Lower magnification (a), and higher magnification (b, c) SEM images of the as-deposited YSZ nanoparticles by EPD during 60min on electropolished steel. 


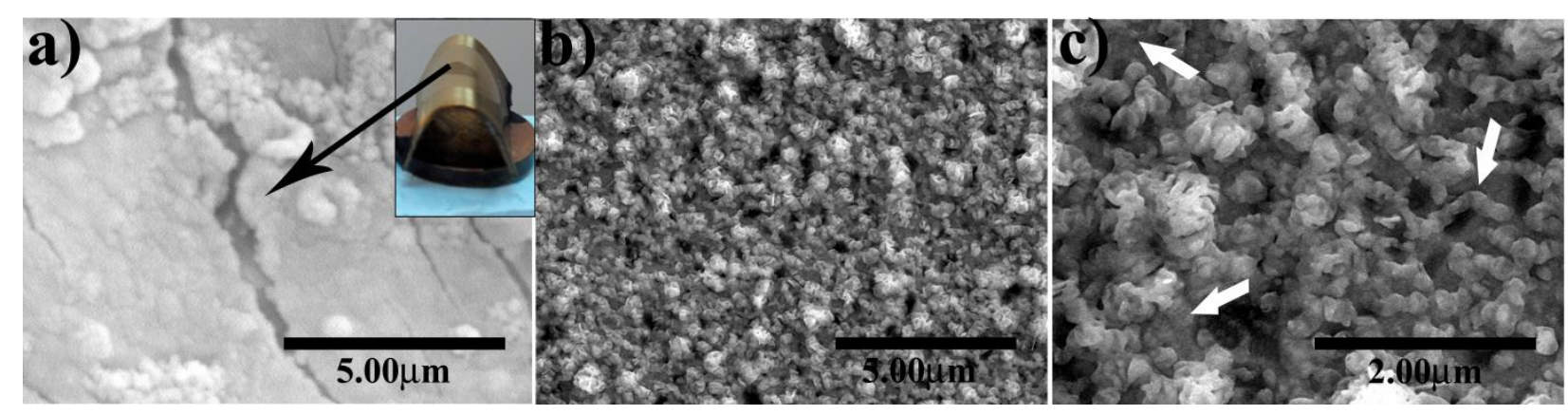

Figure 11. SEM images at different magnifications of the film deposited during 60min on electropolished steel and calcined in air at $500^{\circ} \mathrm{C} / 30 \mathrm{~min}$ with heating and cooling rates of $5^{\circ} \mathrm{C} / \mathrm{min}$. Micrographs show the consolidation degree of the YSZ microstructure after the heat treatment. White arrows (c) point out the microstructure underneath the nanoparticulated surface determined by AFM. 